\title{
The reduction of Crosstalk in VLSI due to parallel bus structure using Data Compression Bus Encoding technique implemented on Artix 7 FPGA Architecture
}

\author{
${ }^{1}$ Syamala Yarlagadda, ${ }^{2}$ Srilakshmi Kaza, ${ }^{3}$ Anil chowdary Tummala, ${ }^{4}$ E Vijaya Babu, ${ }^{5}$ \\ R.Prabhakar \\ ${ }^{1,2}$ Gudlavalleru Engineering College, Gudlavalleru, ${ }^{3}$ Research scholar, VIT-AP, ${ }^{4}$ Vardhaman College of \\ Engineering, Hyderabad, \\ ${ }^{5}$ Mahaveer Institute of Science \& Technology,Hyderabad
}

\begin{abstract}
In this work, a bus encoding method is proposed that reduces the effect of crosstalk. The crosstalk usually occurs when the data is in parallel communicated. In planar structures, the crosstalk effect is large due to the usage of parallel communication and wide data patterns. In bus technique, the huge amount of wires is laid in equal over a significant time. One way to reduce crosstalk without changing the parallel communicating data lines is to reduce the wideband data patterns so as to reduce the power utilization. The proposed encoding method can minimize the crosstalk by reducing wide data patterns without degrading the performance. The architecture is implemented on Artix 7 FPGA at a $28 \mathrm{~nm}$ technology node. The simulation is done using the HDL tool and the results are compared with the existing FPGA architecture. With the proposed method, the wire density and the power consumption are reduced by $57.4 \%$ and $50 \%$ respectively as compared with existing $45 \mathrm{~nm}$ technologies.
\end{abstract}

Keywords - Data transmission, Crosstalk, bus encoding scheme, power consumption, VLSI, noise, propagation delay

\section{INTRODUCTION}

In telecommunication signals, crosstalk is an undesirable disturbance caused due to the electric or magnetic fields from the neighboring circuits which are known as electromagnetic interference (EMI). This problem also arises in microcircuits inside PCs and audio equipment in a network. The term is moreover applied to optical signals that interfere with each other [1].
Regarding electronics, the signal transmitted from one circuit is coupled with another circuit, which leads to undesirable effects named crosstalk. This is also the problem in speech

Signals leaking from other subscriber connections. If the signal propagation medium is analog the crosstalk effects are reduced by using twisted pair cable [2]. Another alternative is a signal conversion using ADC which is immune to crosstalk. In wireless communication, cochannel interference is the problem associated with crosstalk. During music recording process sound leakage from one instrument into a microphone placed before another instrument establishes the crosstalk. There are several sources to create crosstalk, one among them is due to capacitive coupling between the long length of transmission lines which results in unwanted voltage spikes in the neighboring buses. To minimize Crosstalk in fastest performance processor design using a bus encoding is given in [3]. A single wire has been associated with different types of capacitances where the capacitance ' $\mathrm{C}$ ' is distributed between the wire and ground, and the other is the coupling capacitance $\mathrm{C}_{\mathrm{c}}$ between the wire and its neighboring wires [4]. The $\mathrm{C}_{\mathrm{c}}$ of each wire can be divided into $\mathrm{C}_{1}, \mathrm{C}_{2}, \mathrm{C}_{3}$, and $\mathrm{C}_{4}$ (four types) according to the ' $\mathrm{C}$ ' couple of two wires [5]. Forbidden Free Pattern is used to avoid the crosstalk given in [6]. The crosstalk-aware inservice optical path control system using singlemode multi-core fiber transport network introduced in [7]. The error controlling codes using 65nm CMOS technology and PTL with 
minimal performance parameters, codes based on novel pattern classifications are introduced in [8-9]. In worst-case, the crosstalk due to a large number of transitions in the group of lines is minimized using bus encoding scheme which reduce the redundancy and consumption of power [4]. Layout techniques are introduced to eliminate the problem of crosstalk competition presented in [10]. 14\% of crosstalk is minimized using immune coding given in [11]. The $\mathrm{V}_{\mathrm{DD}}$, GND wires are added to a design layout between every signal wire proposed in [12]. Reducing the bus delay up to $75 \%$ by active shielding method which is a more forceful technique as compared to the passive shielding [13]. The optimal delay is achieved by inserting repeaters or buffers further which helps to minimize crosstalk introduced [14]. A physical design technique using statistical approaches requires accurate layout structure and repeater placement to reduce the average delay in circuit [15]. From literature, it was studied that all these techniques are needful for minimizing the average delay during data transmission using bus encoding method. In this work, a $28 \mathrm{~nm}$ technology generations of FPGA devices are used to reduce crosstalk in data transfer with less power consumption and with low delay. In this paper, an introduction to crosstalk and literature is discussed in Section I. The methodology of encoding and decoding are given in Section II. Section III deals with results and discussions and finally, conclusions are given in Section IV.

\section{Methods of Bus EnCoding AND DeCoding}

II (a). Bus Encoding Methods

In Fig.1,the bus encoding blocks are given, which consists of the counter unit, controller, comparator, and three registers with the size of three bits. As shown in Fig.1, this structure accepts 7-bits input data and compresses them to 3-bit data as outputs in the following steps

- The coupling transitions among the 7- bit lines are identified by the number of zero's and one' s in controller and counter blocks.

- The output of the controller is high based on the maximum number of zero's or one's. The numbers of flips in the data lines are identified by comparing data lines of seven bits with the output line.

- Comparator output is set to high when the number of one's is less than the number of zero's and set to low vice-versa.

In general, the possible best cases are two, either all lines are in '0' or ' 1 ' state. The status of bit flip is stored in three-bit registers. So, the only maximum possible flips are three. If all the three register contents are zero, indicated that there are no bit flips leads to no crosstalk. The position of bit flips is shown in Table I.

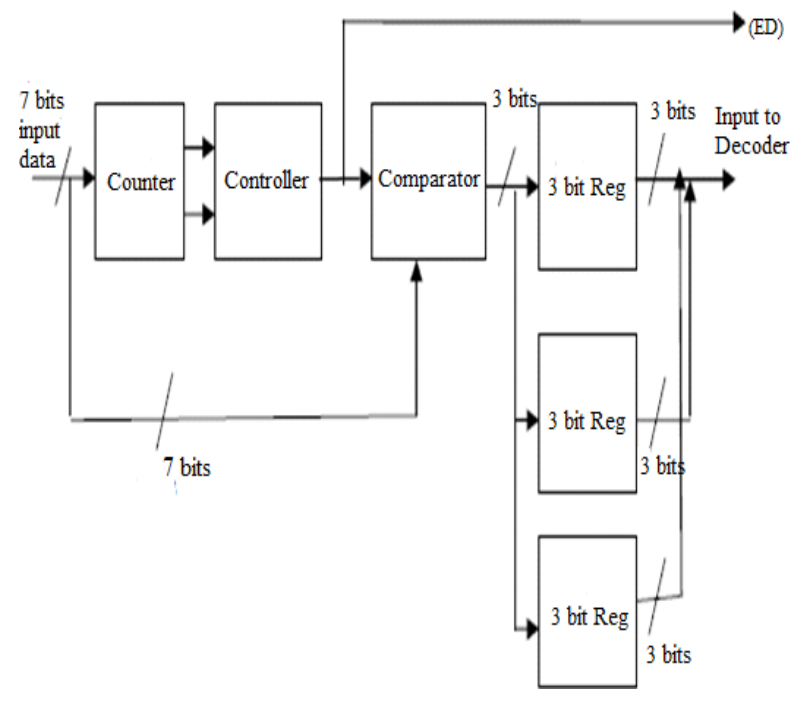

Fig. 1 Structure of encoding scheme

Table I. Flipped Line Positions with respect to Register Content

\begin{tabular}{|l|l|}
\hline $\begin{array}{l}\text { Content of } \\
\text { register }\end{array}$ & $\begin{array}{l}\text { Position of } \\
\text { Flipped Line }\end{array}$ \\
\hline 000 & 0 \\
\hline 001 & 1 \\
\hline 010 & 2 \\
\hline 011 & 3 \\
\hline 100 & 4 \\
\hline 101 & 5 \\
\hline 110 & 6 \\
\hline 111 & 7 \\
\hline
\end{tabular}

The number of zero's and one's are identified by the counter unit in the input pattern by taking seven-bit input. Every input line is selected and compared with zero and one. The controller module is fed with two variables indicating for storing zero's and one' $s$ in the input data. The comparator compares incoming data bit with the 
signal line and identifies the position of flipped bits and store the value in the three-bit register.

\section{II (b). Bus Decoding Methods}

The 3-bit output from the bus encoding logic is given to a 3-bit decoder with enable input as ED as shown in Fig. 2. The internal components of the decoder are three 3-bit registers, splitter, line-identifier, and an inversion module. For each clock cycle, new data is loaded with each of the registers and a three maximum clock cycles are needed for the decoding process. The function of the splitter is to map 1-bit input to 7bits like de-multiplexer. The line to be flipped is identified by line identifier as it takes the data input from three registers. The final decoded output has 7-bits appear at the output after three clock cycles.

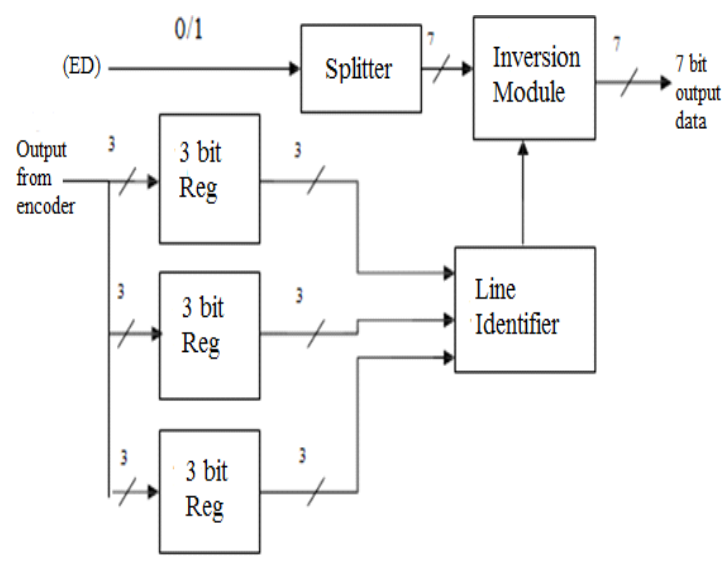

Fig. 2 Block diagram of decoder

\section{RESUlTS AND DISCUSSIONS}

The designed encoder and decoder modules are modeled using HDLs. The functional verification is carried out using Xilinx Vivado and implemented using Artix 7 FPGA at 28nm technology node. Xilinx XPOWER Analyzer is used to get the power consumption of the designed modules for various clock frequencies with different device families of FPGA. Fig. 3(a), shows the functionality of the encoder is depicted. The given input data to the encoder is "0101011" and output obtained as register contents as the state. Similarly, in Fig.3 (b) the functionality of the decoder is given in which the three-bit input "100" is decoded into 7-bit output as "0101011". The combined verification of encoder and decoder is presented in Fig. 4.

\section{A. Simulation Results}

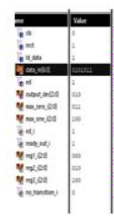

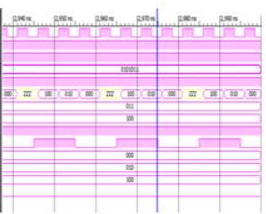

(a)

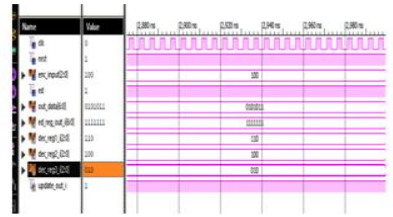

(b)

Fig. 3(a) Simulation Wave form of Encoder (b) Simulation Waveform of Decoder

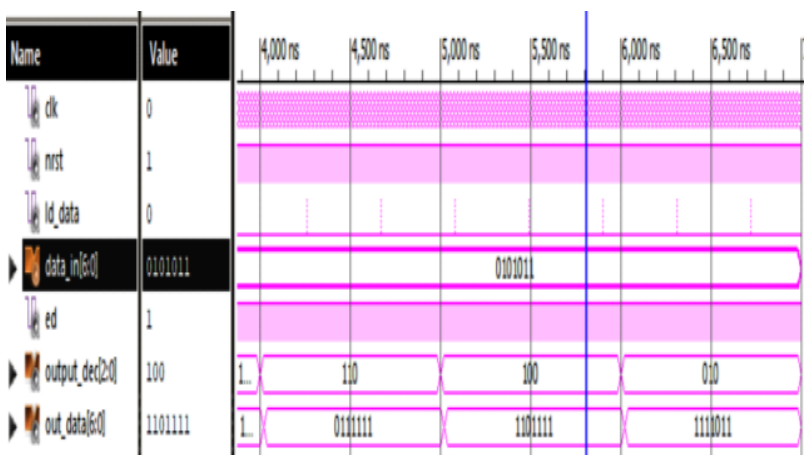

Fig. 5 Simulation Waveform of Encoder-Decoder
The area utilization in terms of the number of logic blocks, IO blocks, timing report of the designed encoder, decoder, and encoder-decoder modules are obtained from the synthesis report. The detailed synthesis reports of the encoder and decoder are given in Table II to Table IV.

\section{B. Synthesis Results}

Table II. Synthesis Report of Encoder

\begin{tabular}{|c|c|c|}
\hline \multicolumn{3}{|c|}{ Device Utilization Summary of Encoder } \\
\hline Devices selected: & $7 \mathrm{7a} 100 \mathrm{tcsg} 324-3$ & \\
\hline \multicolumn{3}{|c|}{$\begin{array}{c}\text { Slice Logic Utilization } \\
\end{array}$} \\
\hline Slice Registers: & 30 utilized out of 126800 & $0 \%$ \\
\hline Slice LUTs: & 51 utilized out of 63400 & $0 \%$ \\
\hline Number used as Logic: & 51 utilized out of 63400 & $0 \%$ \\
\hline \multicolumn{3}{|c|}{ Slice Logic Distribution } \\
\hline LUT Flip Flop pairs used: & 57 & \\
\hline unused Flip Flop: & 27 utilized out of 57 & $47 \%$ \\
\hline unused LUT: & 6 utilized out of & $10 \%$ \\
\hline fully used LUT-FF pairs: & 24 utilized out of & $42 \%$ \\
\hline unique control sets: & 5 & \\
\hline \multicolumn{3}{|c|}{ IO Utilization } \\
\hline IOs: & 14 & \\
\hline bonded IOBs: & 14 utilized out of 210 & $6 \%$ \\
\hline $\begin{array}{l}\text { Specific Feature } \\
\text { Utilization: }\end{array}$ & & \\
\hline Number of & 1 utilized out of 32 & $3 \%$ \\
\hline
\end{tabular}




\begin{tabular}{|c|c|}
\hline BUFG/BUFGCTRLs: & \\
\hline \multicolumn{2}{|c|}{ TIMING REPORT } \\
\hline Total & $0.386 \mathrm{~ns}(0.000 \mathrm{~ns}$ logic, $0.386 \mathrm{~ns}$ \\
& route $)$ \\
& $(0.0 \%$ logic, $100.0 \%$ route $)$ \\
\hline
\end{tabular}

Table III. Synthesis Report of Decoder

\begin{tabular}{|c|c|}
\hline \multicolumn{2}{|c|}{ Device Utilization Summary of Decoder } \\
\hline Selected Device : & 7a100tcsg324-3 \\
\hline \multicolumn{2}{|c|}{ Slice Logic Utilization: } \\
\hline Number of Slice Registers: & $\begin{array}{c}31 \text { utilized out of } 126800 \\
0 \%\end{array}$ \\
\hline Number of Slice LUTs: & $\begin{array}{cc}26 & \text { utilized out of } \\
0 \%\end{array}$ \\
\hline Number used as Logic: & $\begin{array}{cc}26 & \text { utilized out of } 63400 \\
0 \% & \\
\end{array}$ \\
\hline \multicolumn{2}{|c|}{ Slice Logic Distribution } \\
\hline LUT Flip Flop pairs used: & 42 \\
\hline unused Flip Flop: & 11 utilized out of $42 \quad 26 \%$ \\
\hline unused LUT: & 16 utilized out of $4238 \%$ \\
\hline fully used LUT-FF pairs: & 15 utilized out of $4235 \%$ \\
\hline unique control sets: & 7 \\
\hline \multicolumn{2}{|c|}{ IO Utilization } \\
\hline IOs: & 13 \\
\hline bonded IOBs: & 13 utilized out of 210 \\
\hline \multicolumn{2}{|l|}{ Specific Feature Utilization: } \\
\hline BUFG/BUFGCTRLs: & 1 utilized out of 32 \\
\hline \multicolumn{2}{|c|}{ Timing Report } \\
\hline Total & $\begin{array}{c}0.640 \mathrm{~ns}(0.361 \mathrm{~ns} \text { logic, } \\
0.279 \mathrm{~ns} \text { route }) \\
(56.4 \% \text { logic, } 43.6 \% \text { route })\end{array}$ \\
\hline
\end{tabular}

Table IV. Synthesis Report of Encoder-Decoder Device utilization summary of Encoder-Decoder

\begin{tabular}{|c|c|c|}
\hline \multicolumn{3}{|c|}{ Device utilization summary of Encoder-Decoder } \\
\hline Selected Device : & $7 \mathrm{a} 100 \operatorname{tcsg} 324-3$ & \\
\hline \multicolumn{3}{|c|}{ Slice Logic Utilization } \\
\hline $\begin{array}{l}\text { Number of Slice } \\
\text { Registers: }\end{array}$ & 68 utilized out of 126800 & $0 \%$ \\
\hline $\begin{array}{l}\text { Number of Slice } \\
\text { LUTs: }\end{array}$ & 100 utilized out of 63400 & $0 \%$ \\
\hline $\begin{array}{l}\text { Number used as } \\
\text { Logic: }\end{array}$ & 100 utilized out of 63400 & $0 \%$ \\
\hline \multicolumn{3}{|c|}{ Slice Logic Distribution } \\
\hline $\begin{array}{l}\text { Number of LUT Flip } \\
\text { Flop pairs used: }\end{array}$ & 130 & \\
\hline $\begin{array}{l}\text { Number with an } \\
\text { unused Flip Flop: }\end{array}$ & 62 utilized out of 130 & $47 \%$ \\
\hline $\begin{array}{l}\text { Number with an } \\
\text { unused LUT: }\end{array}$ & 30 utilized out of 130 & $23 \%$ \\
\hline $\begin{array}{l}\text { Number of fully used } \\
\text { LUT-FF pairs: }\end{array}$ & 38 utilized out of 130 & $29 \%$ \\
\hline $\begin{array}{l}\text { Number of unique } \\
\text { control sets: }\end{array}$ & 11 & \\
\hline \multicolumn{3}{|c|}{ IO Utilization } \\
\hline Number of IOs: & 17 & \\
\hline $\begin{array}{l}\text { Number of bonded } \\
\text { IOBs: }\end{array}$ & 17 utilized out of 210 & $8 \%$ \\
\hline \multicolumn{3}{|l|}{$\begin{array}{c}\text { Specific Feature } \\
\text { Utilization: }\end{array}$} \\
\hline Number of & 1 utilized out of 32 & $3 \%$ \\
\hline
\end{tabular}

\begin{tabular}{|c|c|}
\hline BUFG/BUFGCTRLs: \\
\hline & TIMING REPORT \\
\hline Total & $\begin{array}{c}0.645 \mathrm{~ns}(0.361 \mathrm{~ns} \text { logic, } 0.283 \mathrm{~ns} \text { route }) \\
(56.0 \% \text { logic, } 44.0 \% \text { route })\end{array}$ \\
\hline
\end{tabular}

The RTL schematic of the encoding and decoding bus is given in Fig. 6. Power analysis is carried out for the designed module at various clock frequencies and families of FPGAs using XPA. The timing path for bus encoding and decoding is obtained as $0.645 \mathrm{~ns}$ targeted to Artix 7 FPGA and verified with different device families and the results are given in Table $\mathrm{V}$

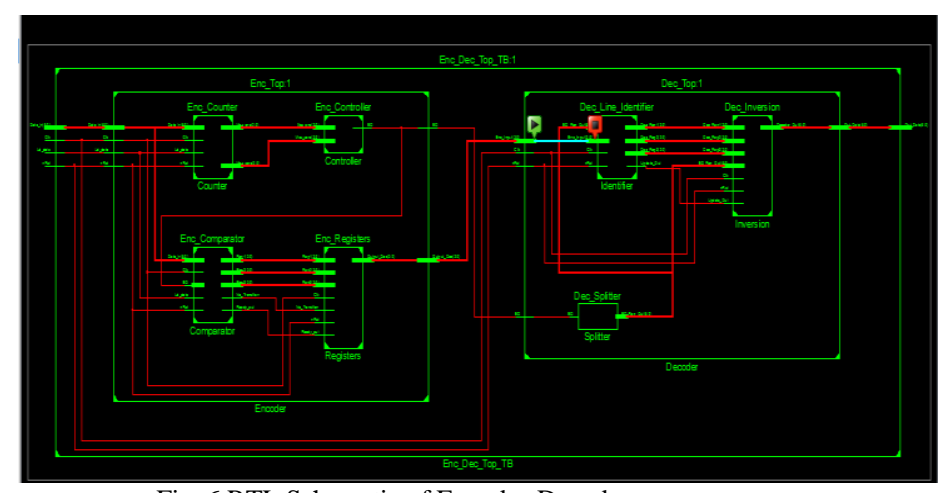

Fig. 6 RTL Schematic of Encoder-Decoder

Table V. Power Consumption, Delay Product and Power Delay Product for Different FPGAs at $500 \mathrm{MHz}$

\begin{tabular}{|l|l|l|l|}
\hline $\begin{array}{l}\text { FPGA } \\
\text { device }\end{array}$ & $\begin{array}{l}\text { Power } \\
\text { Consumption } \\
(\boldsymbol{\mu W})\end{array}$ & Delay(nS) & $\begin{array}{l}\text { Power-Delay } \\
\text { product (f W-S) }\end{array}$ \\
\hline $\begin{array}{l}\text { Spartan6 } \\
\text { Spartan6 }\end{array}$ & 10660 & 5.05 & 53833 \\
$\begin{array}{l}\text { Low power } \\
\text { Virtex7 }\end{array}$ & 9230 & 5.05 & 46611.5 \\
$\begin{array}{l}\text { Virtex6 } \\
\text { Low power }\end{array}$ & 2480 & 0.511 & 3975.58 \\
$\begin{array}{l}\text { Artix7 } \\
\text { (proposed) }\end{array}$ & $\mathbf{5 3 5 0}$ & 0.68 & 16483.2 \\
\hline
\end{tabular}

From Table V, it was observed that Artix 7 consumes less power as compared with other FPGA device families. Note that other than Artix 7 is $45 \mathrm{~nm}$ technology. In highperformance processor applications, the Power Delay Product (PDP) is the most important design metric to indicate the performance of the design which is shown in the same Table $\mathrm{V}$ The amount of consumed power of the proposed bus encoding is further compared with the existing works and is given in Table VI. Finally, it was observed that the encoding method at $28 \mathrm{~nm}$ technology node the consumption of power is lowered as compared with existing works. The obtained results of $28 \mathrm{~nm}$ technology 
bus encoding method have been compared with different techniques used for minimizing crosstalk.[16-19].

Table VI. Power Results of Bus Encoding Method are Compared With Existing Works

\begin{tabular}{|l|l|l|}
\hline Technology & Method & Power(nW) \\
\hline \multirow{4}{*}{$45 \mathrm{~nm}$} & Existing- I [16] & 43375.53 \\
& Existing- II [17] & 40214.31 \\
& LUT-BED[18] & 706397 \\
& LUT-BED-CLA-[19] & 26821 \\
\hline $\mathbf{2 8 n m}$ & Bus Encoding method & $\mathbf{1 0 0 0 0}$ \\
\hline
\end{tabular}

\section{CONCLUSION}

In this work, the bus encoding method has implemented in HDL code.It has done to avoid the crosstalk noise(in decoder). The worst-case crosstalk is significantly eliminates or reduces by using this method. By reducing the number of lines from 7 bits to 3 bits for the output of the encoder crosstalk is minimized. Due to this, the crosstalk effectively minimizes on and average to $50 \%$. In the end, the outputs of both encoding and decoding are the same without any noise. The signal transitions in wires indicate the functionality of the switching and coupling activities which helps to reduce the consumption of power and crosstalk. Finally, it was concluded that the power consumption of $57.4 \%$ is reduced as compared with other encoding methods. Further, this may also vary depending on the chosen style of FPGA.

\section{References}

[1] B.K. Kaushik., S. Sarkar, R.P.Agarwal and R.C. Joshi, "Crosstalk Analysis of Simultaneously Switching Interconnects", International Journal of Electronics, Taylor and Francis (UK), vol. 96, No.10, pp.1095-1114, 2009.

[2] B.K. Kaushik., S. Sarkar, "Crosstalk Analysis for a CMOSGate-Driven Coupled Interconnects", IEEE Trans. on Computer-Aided Design of Integrated Circuits and Systems, vol. 27, No. 6, pp.1150-1154, 2008.

[3] Wen-Wen Hsieh, Po-Yuan Chen, Chun-Yao Wang, and TingTing Hwang, "A Bus-Encoding Scheme for Crosstalk Elimination in High-Performance Processor Design", IEEE Transactions on computer aided design of Integrated circuits and systems, vol. 26, Issue. 12, pp. 2222-2227, January 2008.

[4] Y. Aghaghiri, F. Fallah and M. Pedram, "Transition reduction in memory buses using sector based encoding techniques", IEEE Transactions on Computer-Aided Design of Integrated Circuits and Systems, vol. 23, No. 8, 2004, pp.1164-1174.

[5] Y. Shin, S. Chae and Choi K., "Partial Bus-Invert Coding for Power Optimization of System Level Bus", Proceedings of the International Symposium on Low Power Electronics and Design, pp.127-129, 1998.
[6] R. Srivasavi, A.S. Rao, and P. Srinivas, "Forbidden Free Pattern Crosstalk Avoidance", International Journal of Computer and Communication Technology, vol.3, No.4, 2012.

[7] B.K. Kaushik, S. Sarkar., R.P. Agarwal and Joshi R.C., "Effect of Line Resistance and Driver Width on Crosstalk in Coupled VLSI Interconnects.", Microelectronics International, Emerald Pub., U.K., vol. 24, No. 3, pp. 42-45, 2007.

[8] Z. Khan, T. Arslan and A.T. Erdogan, "A Low Power System on Chip Bus Encoding Scheme with Crosstalk Noise Reduction Capability", IEEE Proc. Computer and Digital Techniques, 2005.

[9] O. Battari and S. Rao, "On chip crosstalk delay and noise analysis using static timing analysis on Nano time ultra in VLSI circuits", Global Journal of Advanced Engineering Technologies, pp.166-172, 2014.

[10] Ashok Vittal, Lauren Hui Chen, Malgorzata MarekSadowska, Kai-Ping Wang, and Sherry Yang, " Crosstalk in VLSI Interconnections", IEEE transactions on computeraided design of integrated circuits and systems, vol. 18, No. 12, pp.1817-1824, December 1999.

[11] J.Venkateswara Rao and A.V.N.Tilak, "A Bus Encoding to Reduce Crosstalk Noise Effect In System On Chip", International Journal of VLSI design \& Communication Systems (VLSICS), vol.2, No.2, pp.110-118, June 2011.

[12] S.P. Khatri, A. Mehrotra, R.K. Brayton, Ralf H.J.M. Otten, and A.L. Sangiovanni-Vincentelli., "A novel vlsi layout fabric for deep sub-micron applications", in Proceedings of Design Automation Conference, IEEE, pp. 491-496, 1999.

[13] C. Duan and S.P. Khatri, "Exploiting crosstalk to speed up on-chip buses", in Proceedings of Design Automation, and Test in Europe (DATE) Conference, 2004.

[14] M. Ghoneima and Y. Ismail., "Optimum positioning of interleaved repeaters in bidirectional buses", Transactions on Computer-Aided Design, vol. 24, Issue 3, pp. 461-469, 2005.

[15] CN. Satyanarayana, M. Mutyam, and A.V. Babu, "Exploiting On-Chip Data Behavior for Delay Minimization", In Proceedings of International Workshop on System-Level Interconnect Prediction, Austin, Texas, USA, IEEE/ACM, 2007.

[16] O. Battari and S. Rao, "On chip crosstalk delay and noise analysis using static timing analysis on Nano time ultra in VLSI circuits", Global Journal of Advanced Engineering Technologies, pp.166-172, 2014.

[17] Z. Shirmohammadi, F. Mozafari, and S.G. Miremadi, "An efficient numerical-based crosstalk avoidance codec design for NoCs", Microprocessors and Microsystems, vol. 50, pp.127-137, 2017.

[18] F. Shi, X. Wu, and Z. Yan, "New crosstalk avoidance codes based on a novel pattern classification", IEEE Transactions on Very Large Scale Integration (VLSI) Systems, vol.21, No.10, pp.1892-1902, 2013.

[19] Battari Obulesu, Parvathaneni Sudhakara Rao, "ASIC Implementation of Low Power Efficient Crosstalk Analytical by LUT-BED-CLA", International Journal of Intelligent Engineering and Systems, vol. 11, No.5, pp. 125-139, 2018. 